Ann. Génét. Sél. anim., I975, 7 (I), I-II.

\title{
HYBRIDATION ARTIFICIELLE \\ ENTRE LA TRUITE ARC-EN-CIEL (SALMO GAIRDNERI RICHARDSON) ET LE SAUMON COHO (ONCORHYNCHUS KISUTCH WALBAUM)
}

\author{
B. CHEvassus et J. PETIT \\ avec la collaboration technique de Anne-Marie ESCAFrRE \\ Laboratoire de Physiologie des Poissons, \\ Centre national de Recherches zootechniques, I. N.R. A., \\ 78350 Jouy en Josas
}

\section{RÉSUMÉ}

Une hybridation entre le Saumon coho mâle (Oncorhynchus kisutch walbaum) et la Truite arc-en-ciel (Salmo gairdneri RICHARDSON) a été réalisée artificiellement, le témoin étant obtenu par croisement de ces mêmes femelles avec des mâles arc-en-ciel. Une estimation du taux moyen de fécondation (mesuré au stade œillé) est de 42,8 p. Ioo et 57,I p. roo dans deux séries d'expériences, contre $93,0 \mathrm{p}$. roo et $94,4 \mathrm{p}$. Ioo pour le témoin (différence significative). Le taux d'éclosion est de $88,4 \mathrm{p}$. Ioo pour l'hybride contre $98,4 \mathrm{p}$. Ioo pour le témoin (différence significative). La durée de développement embryonnaire des hybrides est intermédiaire entre les valeurs des deux parents (445 degré-jours pour l'hybride, 320 degré-jours pour la Truite arc-en-ciel, 472 degré-jours pour le Saumon coho).

Chez l'hybride, ces trois paramètres présentent une importante hétérogénéité interlots, le témoin étant homogène. L'analyse de cette variabilité montre qu'elle est principalement d'origine maternelle.

L'hybridation inverse a également été tentée, mais aucun œuf n'a atteint le stade œillé.

Les problèmes du choix des reproducteurs adéquats pour l'hybridation et de la parenté phylogénétique entre les deux espèces hybridées sont discutés.

\section{I. - INTRODUC'TION}

Depuis ROOSEVELT (I880) de nombreux cas d'hybridation artificielle ou naturelle ont été rapportés chez les Salmonidés. En ce qui concerne la Truite arc-en-ciel (Salmo gairdneri RICHARDSON) les résultats sont cependant très limités, que ce soit 
en hybridation intra ou intergénérique (tabl. I). Dans le sens mâle arc-en-ciel $\times$ femelle hétérologue, le développement embryonnaire n'atteint généralement pas le stade œillé. Dans le sens mâle hétérologue $\times$ femelle arc-en-ciel, un taux d'éclosion important a parfois été observé, mais la survie ultérieure s'est avérée extrêmement faible (Suzuki et Fukuda, I97I ; SEguin, I957 ; Buss, I956 ; Crossman et Buss, I966). Seul l'hybride avec Salmo clarki, espèce très voisine, a montré un taux de survie notable et s'est révélé fertile (Gould, I966 ; CARLANDER, I969 ; NEEDHAM et GARD, I959).

TABLEAU I

Possibilité d'hybridation artificielle de quelques salmonidés avec la Truite arc-en-ciel

Possibility of artificial hybridization of some salmonides with rainbow trout

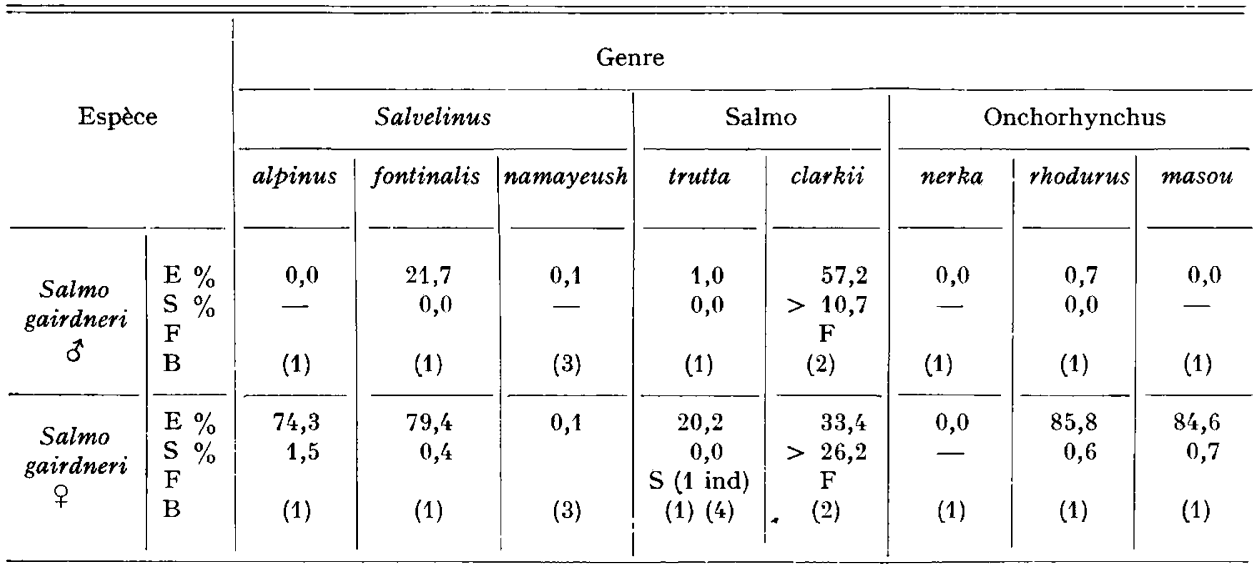

D'après : (1) Suzuki et FukUda (1971).

(2) R. R. Needham et R. Gard (1959)

(3) L. R. Seguin (1957)

(4) K. Buss et J. R. Wright (1958).

$\mathrm{E} \%$ : Pourcentage d'éclosion (rate of hatching)

$\mathrm{S} \%$ : Pourcentage de survie à un an (rate of survival after 1 year)

$\mathrm{F} \quad$ : Fertilité des hybrides $(\mathrm{F}=$ Fertile, $\mathrm{S}=$ Stérile) (Fertility of the hybrids)

B : Références bibliographiques (bibliography).

Cependant, un certain nombre de croisements n'ont pas, à notre connaissance, été décrits. En particulier dans le genre Oncorhynchus qui, selon MrLNE (I948) et NEAVE (I958) dérive du genre Salmo, on considère (MILNE, I948; HoAR, I958) que le genre Saumon coho (Oncorhynchus kisutch WALBAUM) représente une forme primitive, passant encore une partie importante de sa vie en eau douce, alors que les espèces plus évoluées de ce genre, telle le Saumon pink (Oncorhynchus gorbuscha WALBAUM) n'y passent que quelques semaines. A cette parenté phylogénétique et écologique entre la Truite arc-en-ciel et le Saumon coho s'ajoute 1'identité de leurs nombres chromosomiques $(2 n=60$; Simon, I963; BUNGERBERG DE JONG, 1955), 
conditions nécessaires à la réalisation d'une méîose équilibrée chez les hybrides (SvÄRDSON, I945).

Il était donc intéressant d'étudier la possibilité d'hybridation entre ces deux espèces.

\section{II. - MATÉRIEL ETT MÉTHODES}

Les différents croisements ont été réalisés pendant l'hiver 1974 au laboratoire de Physiologie des Poissons à l'I. N. R. A. à Jouy en Josas (Yvelines). Les saumons coho, âgés de 2 ans ont été élevés en eau douce à la pisciculture d'Yvias (Côtes du Nord).

Leurs gamètes ont été prélevés sur place, puis transportés dans la glace fondante et utilisés 7 à 8 heures plus tard. Les gamètes de truites arc-en-ciel provenant de différentes piscicultures françaises (tabl. 2) ont été prélevés juste avant l'insémination artificielle. Pour chaque croisement, 200 à 300 œufs d'une même femelle ont été fécondés à sec par o, $5 \mathrm{cc}$ de laitance, puis additionnés de ro cc de dilueur d'insémination (Petit et al., 1973). Après $20 \mathrm{mn}$, les œufs ont été mis à incuber à $8^{\circ} \mathrm{C}$.

\section{TABLEAU 2}

Origine des reproducteurs utilisés

Origin of the rainbow trout broodstok used in the experiment

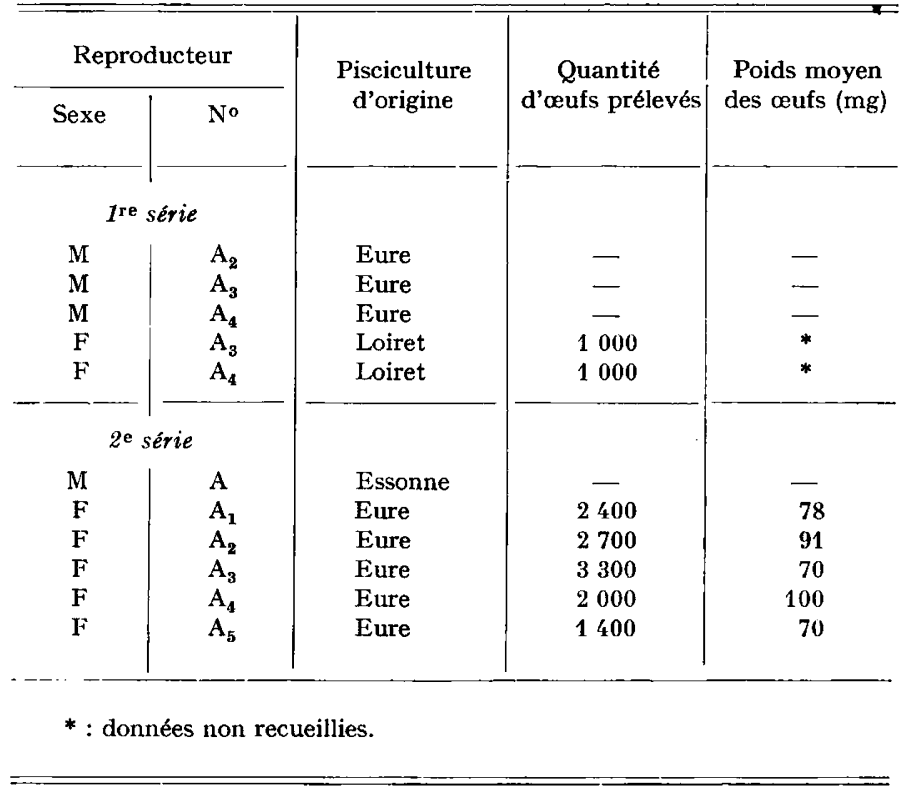

Dans la première série de croisements, nous disposions d'œufs et de sperme de Saumon coho. Les deux croisements témoins et les deux croisements hybrides ont donc pu être réalisés.

Dans la seconde série de croisements, seuls les mâles coho pouvaient être utilisés. Nous avons donc réalisé un plan factoriel complet de 7 mâles coho $\times 5$ femelles arc-en-ciel, chaque femelle arc-en-ciel étant en outre testée par un mâle arc-en-ciel pour fournir le témoin.

Le pourcentage de fécondation a été estimé par le nombre d'œufs atteignant le stade œillé 
rapporté au nombre d'œufs initial, le pourcentage d'éclosion par le nombre d'alevins éclos rapporté au nombre d'œufs œillés.

La durée moyenne du développement embryonnaire exprimée en degré-jours (DJ) (nombre de jours d'incubation $\times$ température moyenne en degré Celsius) a été déterminée par analyse probit de la courbe d'éclosion (FINNEY, I962). Cette courbe est obtenue par comptage biquotidien des œufs éclos. La période d'éclosion est le temps compris entre 2,5 p. I00 et 97,5 p. 100 d'éclos. Les analyses de variance des pourcentages $P$ ont été réalisées sur les données transformées $\mathrm{Y}(\mathrm{Y}=\operatorname{Arc} \sin \sqrt{\mathrm{P} / \mathrm{roo}})$ en modèle additif à effet fixe. L'estimation des données manquantes a été effectuée par la méthode de SNEDECOR (1956) et le test d'effet d'un facteur est le test F de Fischer.

\section{TABLEAU 3}

Principaux résultats enregistrés dans les lots hybrides et témoins

Main results obtained in the hybrids and control crossing groups

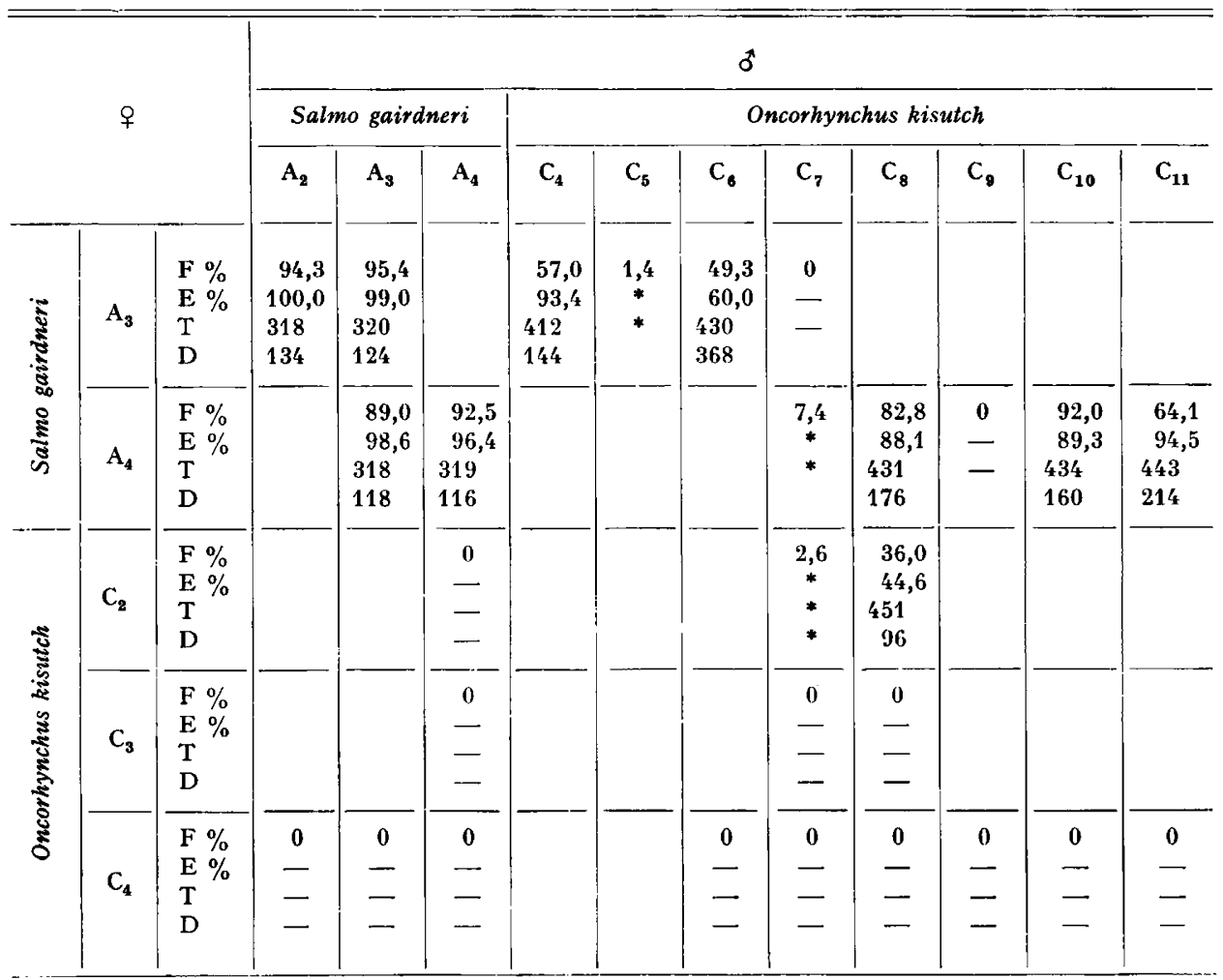

F \% : Pourcentage de fécondation [(nombre d'œufs œillés/nombre d'œufs initial) $\times 100$ ] (rate of fecondation [(number of eyed eggs/initial number of eggs) $\times 100])$.

E \% : pourcentage d'éclosion [(nombre d'individus éclos/nombre d'œufs œillés) $\times 100$ ] (rate of hatching [(number of hatching eggs/number of eyed eggs) $\times 100])$.

D : durée d'éclosion en heures (temps pour avoir 95 p. 100 d'éclos). (period of hatching in hours (time to have 95 p. 100 of hatching eggs)).

$\mathrm{T}: \quad$ temps de développement embryonnaire (en degré-jours) (time of embryonnary development (no of days of incubation $\times$ mean temperature ${ }^{\circ} \mathrm{C}$ )).

* : valeur non déterminée (effectif insuffisant) (undetermined data (small sample)). 


\section{III. - RÉSULTATS}

A. - Production des hybrides et des témoins (tabl. 3)

\section{Mâle arc-en-ciel $\times$ femelle arc-en-ciel.}

Pour les lots témoins arc-en-ciel, le pourcentage moyen de fécondation est de 93,0 p. roo et le taux moyen d'éclosion de 98,4 p. Ioo. Le temps de développement embryonnaire, $3 \mathrm{I} 9 \pm 2 \mathrm{DJ}$ (fig. I) correspond aux valeurs classiquement citées (EMmodx, I934).

Les différents lots se révèlent homogènes pour ces paramètres (tests $\chi^{2}$ non significatifs). On peut donc conclure à une bonne qualité des œufs et des spermes utilisés.

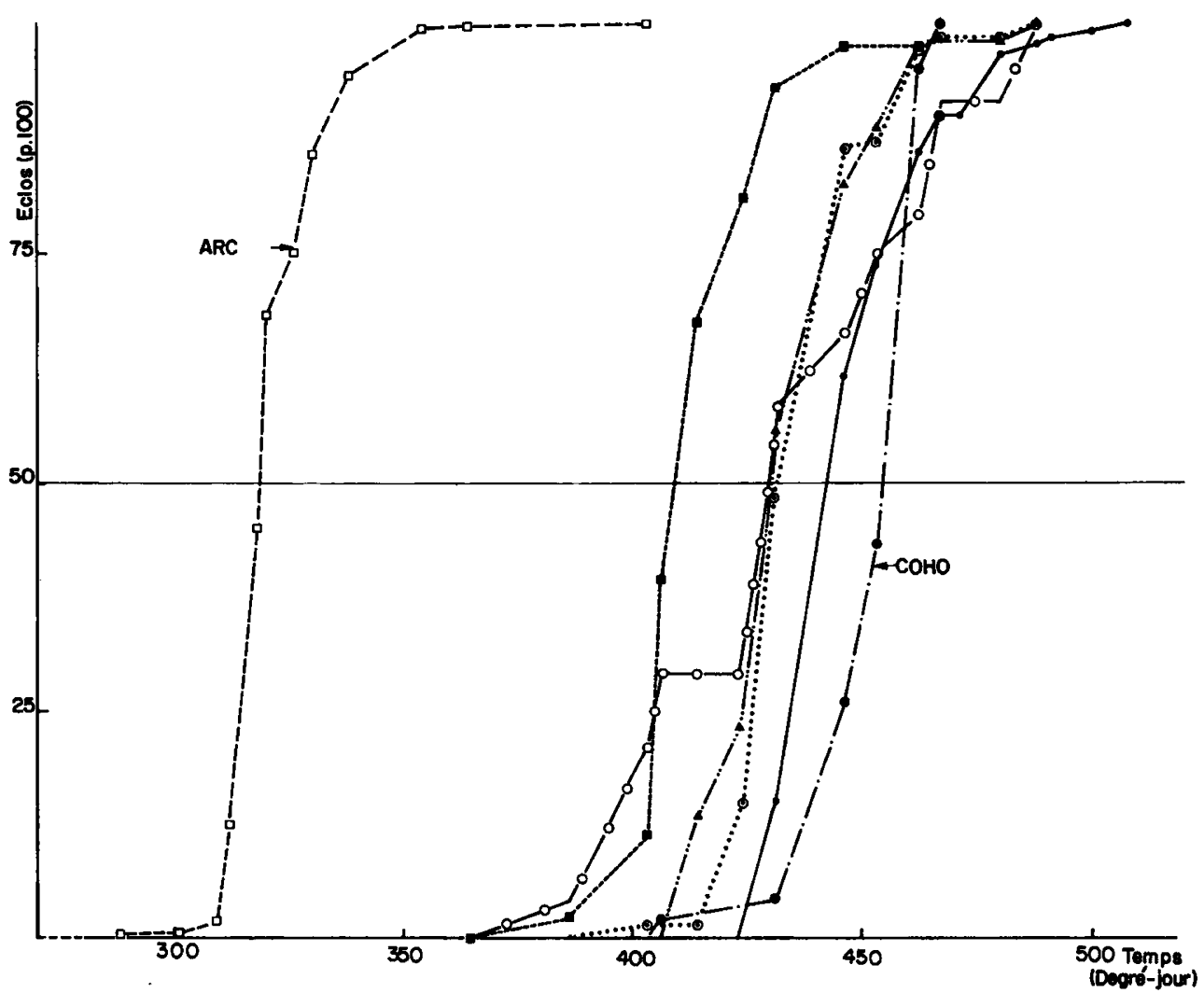

FIg. I. - Courbes d'éclosion

Curve of hasching

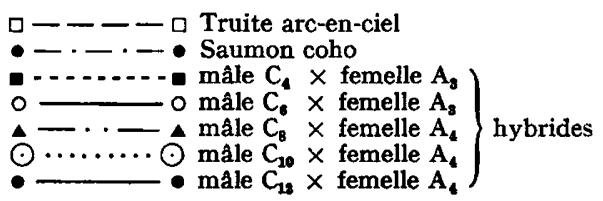


2. Male coho $\times$ femelle coho.

Ces mêmes paramètres mesurés chez le témoin coho sont au contraire extrêmement mauvais. Sur les 6 femelles testées, une seule permet d'obtenir des embryon atteignant le stade œillé, avec un taux de fécondation assez faible (36,0 p. Ioo). Le taux d'éclosion de ces œufs est également mauvais (44,6 p. roo), de nombreux embryons avortant entre le stade œillé et l'éclosion. Le temps de développement embryonnaire (fig. I), déterminé sur un seul lot, est de $45 \mathrm{I} \pm 7 \mathrm{DJ}$.

3. Mâle arc-en-ciel $\times$ femelle coho.

Dans ce croisement aucun œuf n'atteint le stade œillé. L'utilisation du liquide de Stockard 8 jours après la fécondation ne permet de distinguer aucun embryons constitué.

\section{TABLEAU 4}

Analyse de la variabilité d'origine paternelle et maternelle

Analysis of the variability of maternal and paternal origin

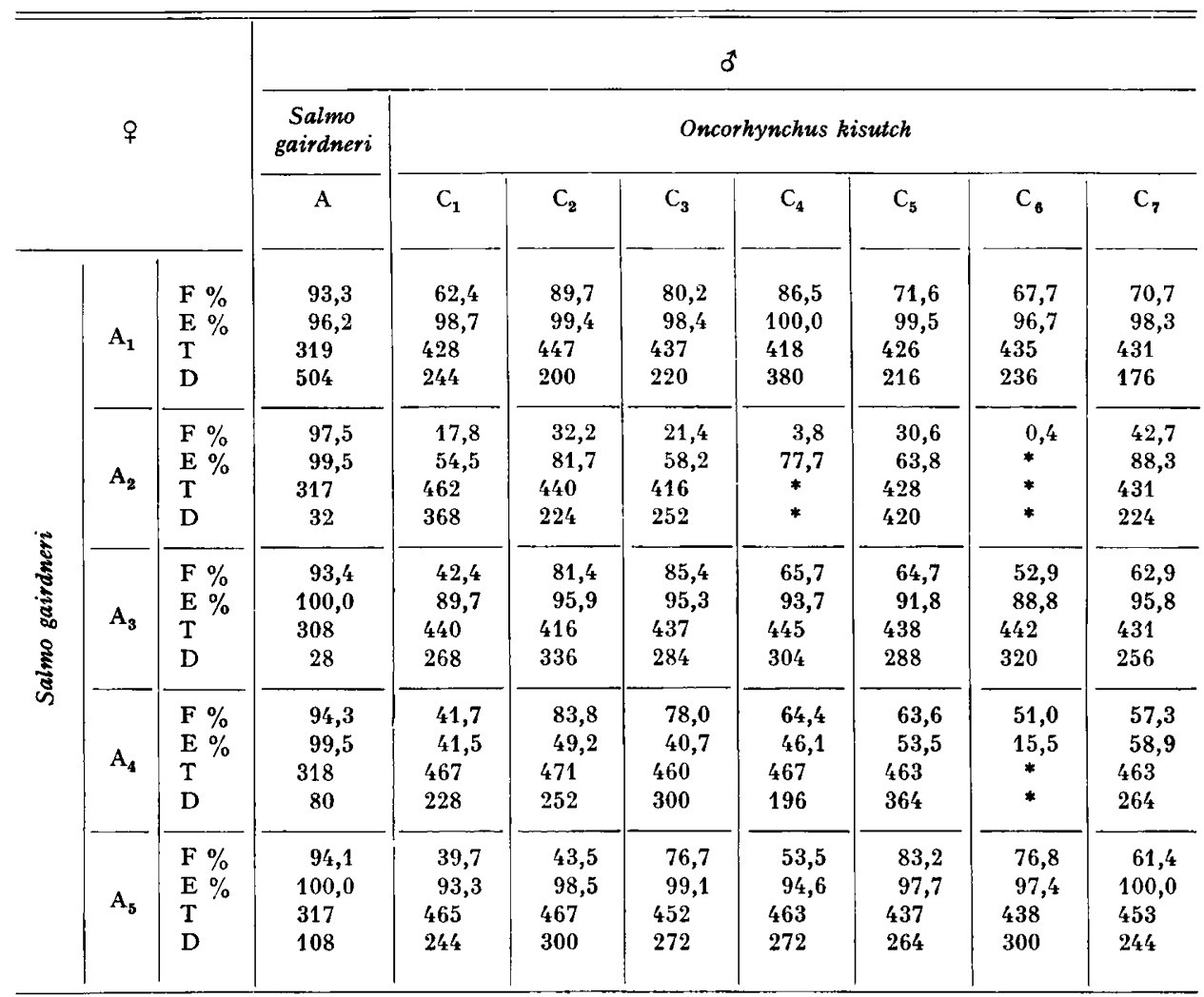

Légende : voir tableau 3 (legend : see table 3). 
4. Mâle coho $\times$ femelle arc-en-ciel.

Dans ce croisement le stade œillé est atteint dans la plupart des lots. Cependant le pourcentage de fécondation est extrêmement différent d'un lot à l'autre : $\left(\chi^{2}\right.$ intermâles $=103,27(3 \mathrm{DL}) ; \chi^{2}$ interfemelles $=34,09$ (I DL) hautement significatif), variant de 0,0 à $92,0 \mathrm{p}$. Ioo avec une moyenne de $42,8 \mathrm{p}$. Ioo.

Le pourcentage d'éclosion varie également $\left(\chi^{2}\right.$ total $=38,44(4 \mathrm{DL})$ hautement significatif, mais est en général satisfaisant $(88,4$ p. Ioo de moyenne).

Le temps de développement embryonnaire (fig. I) est compris entre celui des deux espèces parentales, mais la période d'éclosion est sensiblement plus longue pour 1'hybride (I44 à 368 heures) que pour 1'arc (I24 heures) et le coho (96 heures).

$$
\text { B. - Analyse de variabilité (tab1. 4) }
$$

Le dispositif utilisé pour la seconde série de croisement permet de décomposer la variabilité observée en :

- une variabilité due aux mâles coho;

- une variabilité due aux femelles arc-en-ciel.

\section{Fécondation.}

Pour les hybrides, le pourcentage de fécondation est toujours variable $(0,4$ à 89,7 p. Ioo). L'analyse de variance (tabl. 5) dégage un effet significatif du mâle utilisé et surtout un effet femelle hautement significatif.

\section{TABLEAU 5}

Analyse de variance des effets parentaux

Variance analysis for the parental effects

\begin{tabular}{|c|c|c|c|c|c|}
\hline & Source de variation & $\begin{array}{c}\text { Somme } \\
\text { des carrés }\end{array}$ & $\mathrm{DL}$ & Carré moyen & $F(1)$ \\
\hline Fécondation & $\begin{array}{l}\text { entre femelles } \ldots \ldots \ldots \\
\text { entre mâles } \ldots \ldots \ldots \ldots \\
\text { résiduelle } \ldots \ldots \ldots \\
\quad \text { Total } \ldots \ldots \ldots \ldots\end{array}$ & $\begin{array}{ll}5 & 316,50 \\
1 & 259,21 \\
1 & 794,29 \\
8 & 370,00\end{array}$ & $\begin{array}{r}4 \\
6 \\
24 \\
34\end{array}$ & $\begin{array}{r}1329,12 \\
209,87 \\
74,79 \\
-\end{array}$ & $\begin{array}{c}17,78^{* *} \\
2,81^{*}\end{array}$ \\
\hline Éclosion & $\begin{array}{l}\text { entre femelles } \ldots \ldots \ldots \\
\text { entre mâles } \ldots \ldots \ldots \ldots \\
\text { résiduelle } \ldots \ldots \ldots \ldots \\
\text { Total } \ldots \ldots \ldots \ldots\end{array}$ & $\begin{array}{r}9163,94 \\
447,32 \\
727,91 \\
10339,17\end{array}$ & $\begin{array}{r}4 \\
6 \\
23 \\
33\end{array}$ & $\begin{array}{r}2290,88 \\
74,55 \\
31,65 \\
-\end{array}$ & $\begin{array}{c}72,39^{* *} \\
2,36^{*}\end{array}$ \\
\hline $\begin{array}{c}\text { Durée } \\
\text { développement } \\
\text { embryonnaire }\end{array}$ & $\begin{array}{l}\text { entre femelles } \ldots \ldots \ldots \\
\text { entre mâles } \ldots \ldots \ldots \ldots \\
\text { résiduelle } \ldots \ldots \ldots \ldots \\
\quad \text { Total } \ldots \ldots \ldots \ldots\end{array}$ & $\begin{array}{r}5176,80 \\
853,90 \\
2551,60 \\
8582,30\end{array}$ & $\begin{array}{r}4 \\
5 \\
19 \\
28\end{array}$ & $\begin{array}{r}1294,20 \\
170,78 \\
134,30 \\
-\end{array}$ & $\begin{array}{l}3,64 * * \\
1,27 \mathrm{NS}\end{array}$ \\
\hline
\end{tabular}

(1) ** : Significatif à $\mathrm{P}=0,01$

* : Significatif à $\mathrm{P}=0,05$

NS : Non significatif. 
B. Chevassus, J. PETIT

Cette variabilité due à la femelle n'est pas décelable dans les croisements témoins avec un mâle arc-en-ciel ( $\chi^{2}$ interfemelles $=4,65$ (4 DL) non significatif), qui sont tous satisfaisants (93,3 à 97,5 p. roo).

Du fait de cette variabilité la comparaison des rendements moyens $(57, \mathrm{I}$ p. roo pour l'hybride, 94,4 p. Ioo pour l'arc-en-ciel) est d'une interprétation difficile.

\section{2. Éclosion.}

La réussite de l'éclosion ne semble pas liée à celle de la fécondation (corrélation : $r=+0,28(32 \mathrm{DL})$ non significatif). L'analyse de variance révèle cependant un effet hautement significatif de la femelle arc-en-ciel dans la réussite de l'éclosion (tabl. 5).

Cet effet maternel, qui n'est visible qu'en hybridation, semble corrélé négativement à la taille des ovules émis (corrélation $r=-0,9$ (3 DL) significatif) (fig. 2). Pour les femelles à petits œufs $\left(A_{1}, A_{3}, A_{5}\right)$ les pourcentages d'éclosion sont équivalents en hybridation (96,3 p. Ioo) et en croisement témoin ( 98,9 p. Ioo).

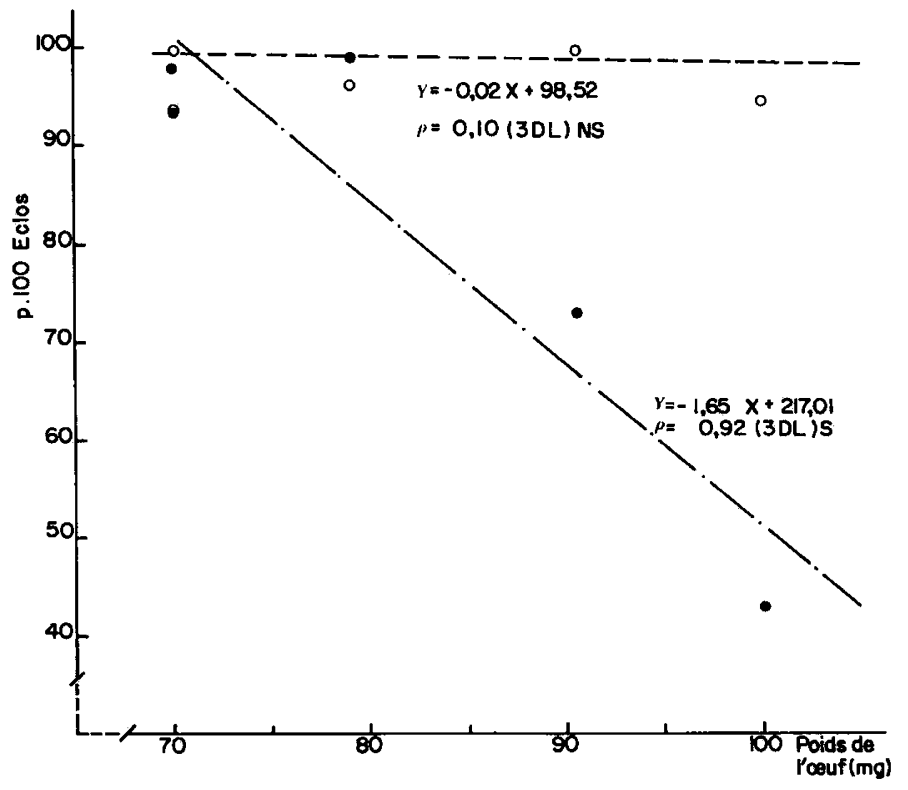

FIG. 2. - Effet de la taille des ceufs

Effect of egg size

o- - o Truite arc-en-ciel

- - - hybride

\section{Temps de développement embryonnaire.}

On observe chez l'hybride une importante variabilité du temps de développement embryonnaire (4I6 à 47I DJ Moyenne $445 \mathrm{DJ}$ ). Ces temps sont supérieurs à ceux du témoin arc-en-ciel (308 à $3 I_{9} \mathrm{DJ}$ ) et, dans la plupart des cas, inférieurs à celui du témoin coho (45 $\mathrm{I} \mathrm{DJ}$ déterminé dans la première série d'hybridations ; 472 DJ selon ALLEN, I958). 
L'analyse de variance (tabl. 5) révèle un effet maternel hautement significatif, qui cette fois ne semble pas lié à la taille des œufs (corrélation $=+0,45$ (3 DL) non significatif). On retrouve également une période d'éclosion généralement plus longue pour l'hybride que pour le témoin.

\section{IV. - DISCUSSION ET CONCIUSIONS}

I. L'hybridation entre la Truite arc-en-ciel femelle et le Saumon coho mâle s'est révélée possible et a conduit à des individus éclos viables, avec un rendement néanmoins inférieur à celui des témoins femelles arc-en-ciel. Le témoin mâle coho $x$ femelle coho est quant à lui de médiocre qualité. Deux faits conduisent à imputer surtout aux femelles ce mauvais résultat. D'une part les mâles coho testés sur des œufs de Truite arc-en-ciel se révèlent féconds; d'autre part, SzöL_ösI (communication personnelle) met en évidence la persistance d'une enveloppe folliculaire autour de l'œuf, ce qui peut expliquer le faible taux de fécondation observé.

Il convient de remarquer que la gamétogenèse des géniteurs coho s'est effectuée très précocement (fraie à 2 ans) et entièrement en eau douce, alors qu'en conditions naturelles les géniteurs ne reviennent en eau douce qu'un à trois mois avant la fraie, à l'âge de 3 à 4 ans (HARAche et Boulineau, I97I).

Dans le cas des mâles, ALM (r943) a montré chez le Saumon atlantique que les taux de fécondation obtenus en utilisant comme géniteurs des parrs (animaux de 2 ans) ou des animaux plus âgés ne différaient pas significativement. Par contre l'utilisation des femelles matures à 2 ans se révèle peu satisfaisante chez les Salmonidés d'eau douce (HUET, I970). Ces données vont également dans le sens d'un rôle prépondérant des femelles dans le mauvais rendement observé pour la fécondation dans le témoin coho.

2. Les résultats obtenus dans les lots hybrides sont extrêmement variables, les meilleurs lots étant équivalents au témoin arc-en-ciel. On peut donc penser qu'un choix des reproducteurs donnant les meilleurs résultats permettrait d'améliorer notablement le taux de réussite de l'hybridation, sous réserve d'une éventuelle interaction mâle $\times$ femelle non observable ici.

Cependant, la présence d'un effet maternel important des femelles arc-en-ciel, non décelable dans un croisement témoin avec un mâle arc-en-ciel, pose le problème du choix de ces reproducteurs. La taille des œufs semble jouer un rôle important dans la réussite de l'éclosion, mais d'autres facteurs (vieillissement des ovules avant fécondation, souche) seraient à envisager. Dans cette optique, ALM (I955) observe que 1'hybride Salmo salar femelle $\times$ Salmo trutta mâle est beaucoup plus aisé à obtenir en utilisant un mâle de la variété migratrice (Salmo trutta marina) qu'en utilisant la variété sédentaire (Salmo trutta fario). De même, Suzuki et Fukuda (I97I) obtiennent parfois des résultats différents suivant la provenance géographique ou les caractéristiques des souches utilisées.

3. L'hybride réciproque n'a pu être obtenu. Cependant, la faible fertilité des femelles coho peut suffire à expliquer le phénomène.

4. La durée de développement embryonnaire de 1'hybride est intermédiaire entre celui de deux parents, mais plus proche de l'espèce paternelle (saumon coho). 
La variabilité des dates d'éclosion est plus importante chez 1'hybride que chez les deux parents, que se soit la variabilité interlots (hétérogénéité des temps moyens et de développement embryonnaire) ou intralots (période d'éclosion plus longue).

5. L'existence d'un hybride intergénérique viable pose le problème de la relation phylogénétique entre les deux espèces utilisées. Critiquée par MOORE (I949) et NEAVE (I958), l'étude des hybridations conduit cependant, dans certains cas, à retrouver les parentés phylogénétiques réelles (HESTER, I970). Cependant, l'étude caryologique des espèces voisines (BEHNKE, I970 ; SIMON, I963) montre que Salmo gairdneri ne peut représenter l'ancêtre à partir duquel a divergé le genre Oncorhynchus. Celles-ci (Salmo clarki clarki, Salmo clarki lewisi) apparaissent en effet comme plus primitives, leur nombre de chromosomes et leur nombre fondamental (nombre de bras chromosomiques) étant plus important.

Le schéma serait donc une évolution parallèle des deux genres par réduction du nombre de chromosomes (fusions centriques) et réduction du nombre fondamental (à partir d'un ancêtre commun) (NEAVE, I958; Simon, r963).

6. Les caractéristiques de cet hybride sont maintenant à déterminer, en particuiier la possibilité d'acquisition de la résistance à la SHV, caractère que possède le Saumon coho, mais non la Truite arc-en-ciel (DE KINKELIN, communication personnelle), les effets d'hétérosis éventuels sur la croissance, la fertilité et les possibilités de croisements en retour avec les espèces parentales.

Reçu pour publication en février 1975.

\section{REMERCIEMENTS}

Cette étude a été conduite dans le cadre d'un contrat avec le C. N. E. X. O. (74-10o3) et a été réalisée grâce à la collaboration de M. CALMELs.

\section{SUMMARY}

\section{ARTIFICIAL, HYBRIDIZATION BETWEEN A FEMALE \\ OF RAINBOW TROUT ( $S$. GAIRDNERI) AND A MALE OF COHO SALMO (o. KISUTCH)}

An artificial hybridization between Oncorhynchus kisutch (coho salmon) male and Salmo gairdneri (rainbow trout) female has been performed. As control, some eggs from the same females were fertilised by male rainbow trout.

The average rate of embryonation (mesured to the eyed stage, which give an estimation of the percentage of fertilization) was $42.8 \mathrm{p}$. Ioo and $57.1 \mathrm{p}$. Ioo for the hybrids in two series of experiments and $93.0 \mathrm{p}$. 100 and $94.4 \mathrm{p}$. Ioo for the control.

The rate of hatching was 88.4 p. Ioo for the hybrid and 98.4 p. roo for the control (significative difference).

At $8^{\circ} \mathrm{C}$ the duration of embryogenesis was intermediate between those of the two parents (55.6 days for hybrids, $4^{\text {o. }}$ days for rainbow trout, 59.0 days for coho salmon).

In hybrids, the rate of fertilization and hatching and the lenght of embryonic development were more heterogeneous than in controls. This variability was shown to be from maternal origin.

Hybridization between female coho salmon and male rainbow trout was not successful; the eggs did not reach the eyed stage.

The problem of the choice of the parents to be used in hybridization is discussed and also the phyllogenetic link between the two hybridised species. 


\section{RÉFÉRENCES BIBLIOGRAPHIQUES}

Allen G. H., 1958. Survival through hatching of eggs from Silver Salmon (Oncorhynchus kisutch). Trans. Am. Fish. Soc., 87, 207-219.

Alm G., 1943. Fertilization experiments with Salmon Parr. Medd. statatens undersöknings och försohsanstalt for sötrattens fisket., 22, I-40.

ALM G., 1955. Artificial hybridization between different species of the Salmon family. Rept. Inst. Freshw. Res. Drottninghom, 36, 13-56.

BeHnke R. J., 1970. The application of cytogenetic and biochimical systematics to phylogenetic problems in the family Salmonidae. Trans. Am. Fish. Soc., 99, 237-248.

Bungerberg De JONG C. M., I955. Cytological studies on Salmo irideus. Genetica, 27, 472-483.

Buss K., 1956. Results of species hybridization within the family Salmonidae. The progressive fish culturist, oct. I956.

Buss K., Wright J. E., I958. Appearance and fertility of trout hybrids. Trans. Am. Fish. Soc., 87, I72-I8I.

CARLANDER K. R., 1969. Handbook of freshwater fishery biology, 1, 157. The Iowa State Univ. Press, Ames, Iowa.

Crossman E. J., Buss K., 1966. Artificial hybrid between Kokanee (O. nerka) and brook trout (Salvelinus fontinalis). Copeia (2), 257-259.

ЕмвоDy G. C., I934. Relation of temperature to the incubation periods of eggs of four species of trout. Trans. Am. Fish. Soc., 64, 281-292.

Finney D. J., r962. Probit analysis. Cambridge, Univ. Press.

Gould W. R., 1966. Cutthroat trout $\times$ golden trout hybrids. Copeia (3), 599-600.

Harache Y., Boulineau J. J., I971. L'élevage des Salmonidés migrateurs amphibiotiques en Amérique du Nord. Rapp. Scient. Techn., CNEXO, nº 5 .

Hester F. E., I97o. Phylogenetic relationships of sunfishes as demonstrated by hybridization. Trans. Am. Fish. Soc., 99, 100-104.

HOAR W. S., I958. The evolution of migratory behaviour among juvenil salmon of the genus Oncorhynchus. Biol. Rev. Cambridge phil. Soc., 15, 391-428.

Huet M., 1970. Traité de Pisciculture, p. r ro, Edt. CH. De Wyngaert, Bruxelles.

MILNE D. J., 1948. The growth, morphology and relationship of the species of Pacific Salmon and the Steelhead Trout. MS. Ph. D. Thesis, McGill Univ.

Moore J. A., 1949. Patterns of evolution in the genus Rana. Genstics, paleontology and evolution, Princeton.

NeAve F., 1958. The origin and speciation of Oncorhynchus. Trans. Roy. Soc. Canada, LII, ser III, sec. 5, 25-39.

Needham P. R., Gard R., I959. Rainbow trout in Mexico and California with notes on the cutthroat series. Univ. of California. Pub. Zool., 67, r-124.

Petit J., Jalabert B., Chevassus B., Billard R., r973. L'insémination artificielle de la Truite (Salmo gairdneri RICHARDSON). I. Effet du taux de dilution, du pH et de la pression osmotique du dilueur sur la fécondation. Ann. Hydrobiol., 4, 201-210.

Roosevelt R. B., I88o. Hybrids. Trans. Am. Fish. Cult. Assoc., N. Y.

SEguin L. R., I957. Scientific fish culture in Quebec since r945. Trans. Am. Fish. Soc., 86, r $36-143$.

Simon R. C., rg63. Chromosome morphology and species evolution in the five north American species of Pacific salmon (Oncorhynchus). J. Morphol., 112, 77-97.

Simon R. C., Dollar A., I963. Cytological aspects of speciation in two north American teleosts, Salmo gairdneri and Salmo clarki lewisi. Canad. J. Genet. Cyto., 5, 43-49.

SNedecor G. W., 1956. Statistical methods. The Iowa State Univ. Press, Ames, Iowa.

Suzuki R., FukudA Y., I97I $a$. Survival potential of $F_{1}$ hybrids among Salmonid fishes. Bull. Freshwater Fish. Res. Lab., 21, 69-83.

Suzuki R., Fukuda Y., I97I $b$. Growth and survival of $\mathrm{F}_{1}$ hybrids among Salmonids fishes. Bull. Freshwater Fish. Res. Lab., 21, $1 \times 7-\mathrm{r}_{3} 8$.

Suzuki R., Fukuda Y., 1973. Appearance and numerical characters of $F_{1}$ hybrids among Salmonids fishes. Bull. Freshwater Fish. Res. Lab., 28, 5-32.

Suzuki R., Fukuda Y., r973. Sexual maturity of $\mathrm{F}_{1}$ hybrids among Salmonid fishes. Bull. Freshwater Fish. Res. Lab., 23, 57-74.

SvÄrdson G., 1945. Chromosome studies on Salmonidae. Reports from the Swedish State Inst. of Freshwater Fish. Res., Drottninghom no 25. 